\title{
TEXTURE FREE RADARGRAMMETRIC PROCESSING OF OPPOSITE-VIEW TOMOSAR DATA FOR DEM ESTIMATION
}

\author{
Francesco Banda, Stefano Tebaldini \\ Politecnico di Milano, DEIB
}

\begin{abstract}
In this paper we propose a new methodology to estimate the absolute Digital Elevation Model (DEM) of an area by radargammetric-like processing of interferometric multi-baseline SAR data from two opposite-side surveys. Two separate DEMs of the imaged area obtained from the multi-baseline interferometric phase of two opposite-side tomographic SAR views are coregistered, correcting residual baseline errors. This methodology combines the great accuracy of multi-baseline interferometric processing with precise stereo plotting typical of opposite-side radargrammetry, requires no texture matching and no control points and can be applied also in the case of few a-priori information about the site topography.
\end{abstract}

Key words: Synthetic Aperture Radar Interferometry (InSAR), Radargrammetry, Digital Elevation Model (DEM).

\section{INTRODUCTION}

Retrieving the Digital Elevation Model (DEM) of an area has been one of the very first applications of Synthetic Aperture Radar (SAR) imaging [MFP11]. A DEM can be classically obtained from a pair of SAR acquisitions through either radargrammetry or interferometry. Radargrammetry applies the principles of stereo vision to a pair of intensity SAR images: the value of the disparity between the two images is related to the absolute elevation through acquisition geometry parameters. A wide baseline between the two acquisitions increases the disparity values, making the resulting estimation more accurate. As a case limit we can think of opposite-side configurations, in which the two views are acquired from each side of the scene. In this case, however, the higher sensitivity given by the great baseline separation is often hindered by the geometric and radiometric differences that occur between the two intensity images [MFP11]. SAR Interferometry (InSAR) $\left[\mathrm{RHJ}^{+} 00\right]$, instead, relies on phase differences between a coregistered pair of images acquired from slightly different views to estimate scene elevation. In this way no texture is required and the estimate of scene elevation has a higher accuracy than in the radar- grammetric case with the same configuration, the differential distance measurement accuracy being a fraction of the wavelength. Anyhow, opposite-side configurations are not possible, being the baseline of such configurations much greater than the critical baseline $\left[\mathrm{RHJ}^{+} 00\right]$, which is the separation between the two tracks in the vertical direction corresponding to a total loss of coherence between the passes. SAR Tomography (TomoSAR) [RM00] is an extension of InSAR to the multi-baseline case: with more than two acquisitions of the same scene from slightly different views, different vertical wavenumbers of the object under investigation are illuminated. The reflectivity profile of the scene in the vertical direction and SAR multi-baseline data form a Fourier pair and the former can be reconstructed by using spectral estimation techniques [RM00, GLM02]. With TomoSAR the contribution of surface scattering can be better localized than in the case of InSAR, the whole vertical scene reflectivity is retrieved instead of only the average height of all backscattering contributions within the resolution cell [RM00]. Both InSAR and TomoSAR require a precise phase calibration, in order to correct for baseline errors that produce residual phase screens in SAR interferograms as low-frequency phase modulations [RPM06]. Phase-calibrated InSAR and TomoSAR data can be used to retrieve an absolute DEM of the imaged area from the interferometric phase. Calibration can be achieved exploiting one or more control points. Alternatively, since the availability of control points is often difficult to meet in practice, dedicated InSAR procedures exploiting additional redundant information provided for instance by multi-baseline acquisitions [GTMdR11] or an external DEM $\left[\mathrm{PEB}^{+} 14\right]$ can be employed.

In this paper a new methodology is proposed for absolute DEM estimation of the imaged scene. First, two different DEMs of the area of interest are obtained from two independent opposite-side multi-baseline SAR surveys, as corresponding to the surface layer of the 3D scene. This is done by estimating and removing baseline errors from the InSAR phase [TMdBP13]. The DEMs thus obtained both represent the DEM of the area up to a rotation of the coordinate system used for representing the imaged scene, which is different for every azimuth and between the two views. Such rotations corresponds to the ambiguity of the calibration/localization problem and come from the rotational invariance of sensor-to-target distances. For every azimuth, a pair of rotations can thus be fixed by 
coregistering the DEMs resulting from the opposite views as a function of the pair of correction rotations applied. A final DEM may then be obtained by fusing the two corrected views. The methodology is validated on ice data from ESA IceSAR 2012 [DNKvdW13].

\section{TOPOGRAPHY ESTIMATION WITH DOU- BLE LOCALIZATION}

\subsection{Double Localization Problem}

Phase calibration of multi-baseline SAR data was considered in [TMdBP13]. If we consider a planar (2D) scenario where $N$ sensors and $P$ targets are present the double localization problem is cast in terms of estimating the sensor and target positions in the 2D space based on the set of distances $R_{n}^{p}$ from each sensor to each target. It is immediate to see that the set of distances is invariant with respect to any distance-preserving coordinate transformation. Accordingly, assuming that the position of one reference sensor is known, the solution will be defined up to a rotation of the coordinate system around the reference sensor. Assuming a nominal solution and considering the expression of the phase derivatives about this solution:

$$
d \varphi_{n}^{p}=k_{z, n}^{p} d z^{p}+\frac{4 \pi}{\lambda}\left(-d Y_{n} \sin \theta^{p}+d Z_{n} \cos \theta^{p}\right)
$$

where: $\theta^{p}$ is the nominal incidence angle at $p$; $k_{z, n}^{p}=\frac{4 \pi}{\lambda} \frac{b_{n}^{p}}{R^{p} \sin \theta^{p}}$ is the nominal height-to-phase conversion factor for the $n-t h$ flight at position $p$ [GTMdR11], where $b_{n}^{p}$ is the normal baseline and $\lambda$ is the system wavelength; $d z^{p}$ is the elevation error for the target at position $p ; d Y_{n}$ and $d Z_{n}$ are the baseline errors for the $n-t h$ flight in ground range and elevation. The unknowns in Equation (1) are represented by target elevation error $d z^{p}$ and the baseline errors $d Y_{n}, d Z_{n}$. Assuming that the angular position of one target with respect to the reference sensor is known, Equation (1) corresponds to a linear system of $N_{e}=(N-1) P$ equations in $N_{x}=2(N-1)+P-1$ unknowns. The condition $N_{e} \geq N_{x}$ cannot be verified unless $N \geq 3, P \geq 3$, that is at least 3 sensors are available, and 3 targets as well.

\subsection{Scene Topography Estimation}

As it is described in Section 2.1, the elevation of the scene can be retrieved solving Double Localization problem along a constant azimuth cut, up to a global rotation of the coordinate system around the reference (or master) sensor position. When dealing with a different azimuth cut, a different rotation will correspond to the new problem ambiguity. With no a-priori information to fix residual rotations, consistent elevation values for the entire 3D scenario can be retrieved by constraining continuity between the estimates of baseline errors between consecutive azimuth cuts. The algorithm for elevation estimation of the entire scene is thus formulated as:

1. Estimate scene elevation at starting azimuth location $x_{i}$ and subtract from InSAR phase

2. Estimate baseline errors at location $x_{i}$ and subtract from InSAR phase

3. Iterate points 1-2 until convergence

4. Repeat points 1-3 at location $x_{i+1}$, subtracting the baseline errors estimated at $x_{i}$ from InSAR phase as a starting point

5. Repeat point 4 until the entire scene is covered

The assumption of continuity is indeed consistent with the low-pass nature characterizing variations of the baseline errors [RPM06]. However, residual low frequency undulations may still persist in the estimated surface height as resulting from error propagation in the estimate of baseline errors. The entity of this disturbance is proportional to the total normal baseline aperture. Such fact can be highlighted assuming the linearized model of Equation (1) for the estimation of elevation $z^{p}$ of a target in $p=(x, y, z)$ from multi-baseline interferometric phase. Imposing continuity in the estimate of the baseline errors and considering, for the sake of simplicity, a regular baseline spacing $\left(k_{z, n}^{p}=n K\right)$, the estimate of elevation at location $p_{1}=\left(x_{1}, y, z\right)=(x+d x, y, z)$ can be expressed as:

$$
\hat{z}^{p_{1}}=z^{p_{1}}+\frac{\sum_{n} n\left(\nu_{n}\left(x_{1}\right)-\hat{\nu}_{n}(x)\right)}{K \sum_{n} n^{2}}
$$

where: $\nu(x)$ is the baseline error term including $y$ and $z$ contributions; $K^{2} \sum_{n} n^{2}=\sum_{n}\left(k_{z, n}^{p}\right)^{2}$ and $K$ is a constant term depending on the normal baseline spacing. To simplify the notation, only the dependence of the error term $\nu$ from the specific along-track location $x$ has been made explicit. The recovered elevation will thus be $z^{p_{1}}$ plus an error term depending on baseline error difference between two consecutive $x$ positions. For a fixed number $N$ of passes, the higher the total normal baseline aperture, the higher $K$, thus the lower the contribution of the disturbance to the phase. Conversely, if the baseline aperture is small, the disturbance will impact more on the phase.

\section{REGISTRATION OF TWO OPPOSITE-SIDE MULTI-BASELINE INSAR VIEWS}

Having two opposite-side surveys available, the DEM of the scene in a real world georeferenced coordinate system can be obtained by coregistering the elevation profiles estimated from the two views, affected by rotational 

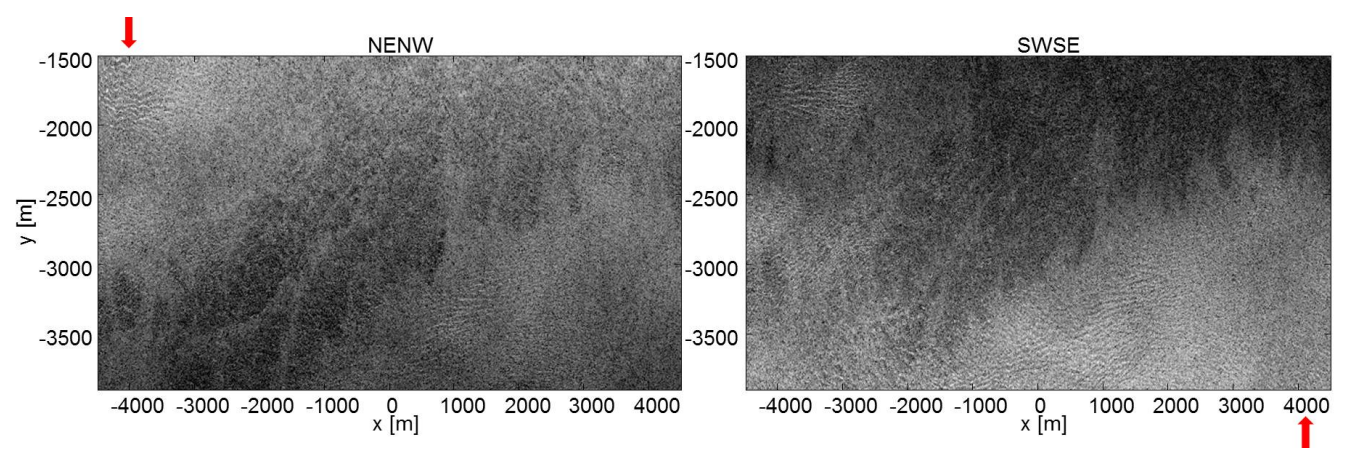

Figure 1. Intensity of master images for NENW (left) and SWSE (right) data in the $x-y$ (azimuth-ground range) plane. Data are represented within the same coordinate frame. Radar look direction for each survey is indicated with a red arrow.
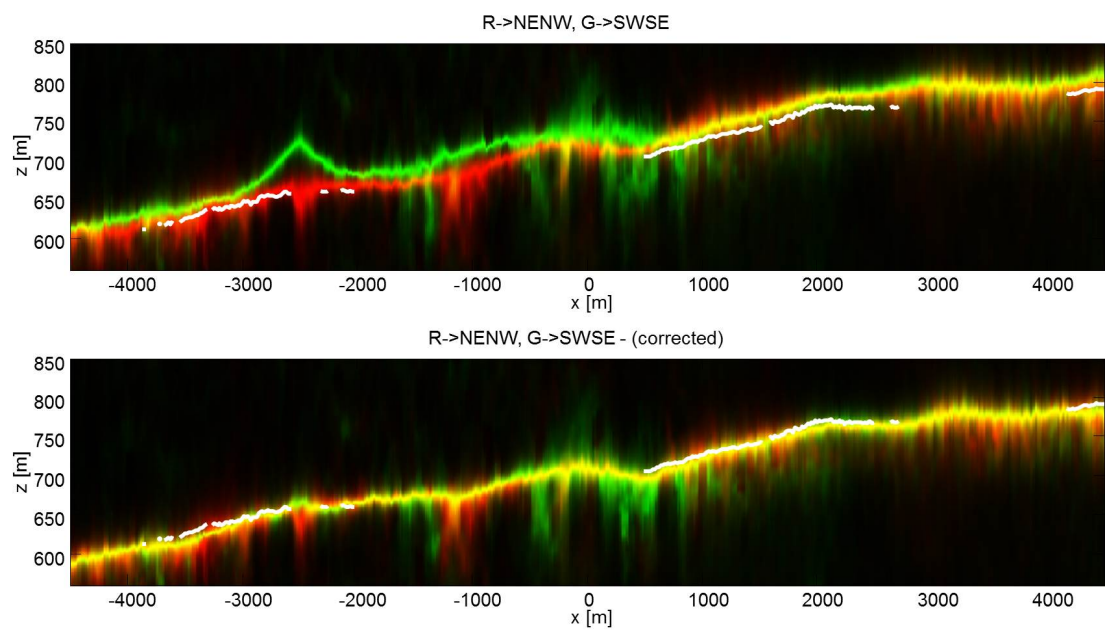

Figure 2. Vertical sections in the $x-z$ plane for NENW data (red) and SWSE data (green), before (upper panel) and after (lower panel) correction of residual rotations. LIDAR DEM is superimposed in white. z axis represents absolute elevations with respect to WGS84 ellipsoid.

ambiguity. This approach is analogous to the matching step performed in radargrammetric processing [MFP11], in which a registration map related to scene elevation can be obtained from patch-wise correlation of an intensity SAR image pair. With respect to radargrammetry however, here no scene texture is required in order to perform coregistration, since elevation profiles relative to the surface layer of the 3D scene obtained from multi-baseline data can be directly compared. Thus, the methodology turns out to be particularly useful in the case of scenes lacking features for the application of techniques requiring textures, like the case of land ice, treated in this paper. The pair of rotations that correctly matches elevation profiles estimated from opposite-side views for each azimuth cut can be fixed according to a suitable chosen metric, for instance by maximizing the correlation between the estimated elevation profiles of the two views. After having corrected the rotations, an absolute DEM can be obtained as the weighted average of the two DEMs resulting from the two opposite-views. Suitable weights for each point may be chosen as the inverse of the tomographic vertical resolution at each location [RM00]. In this way the estimate characterized by the best sensitivity in terms of vertical separability of the targets between the two views will be favored at each location.

\section{EXPERIMENTAL RESULTS}

In this section experimental results are illustrated on P-band HH polarization airborne SAR data of SHR measurement site (approx. $67^{\circ} \mathrm{N}-50^{\circ} \mathrm{E}$ ), on the Russell Glacier, Greenland. Data were acquired on May 7, 2012 by Technical University of Denmark's radar POLARIS in the framework of the European Space Agency IceSAR 2012 campaign [DNKvdW13]. The investigated area is located in the ablation zone of the glacier [CP10] and it has a quite smooth topography, the average altitude is approximately $700 \mathrm{~m}$. Data were specifically acquired for a tomographic experiment by flying POLARIS repeatedly on a nominally straight track, the baselines corresponding in this case to the deviations from the planned track caused by the achievable flight control precision and impact of the wind. This choice guarantees almost unambiguous 3D imaging lacking a-priori knowledge of the 
effective penetration depth and wind conditions. Yet, this represents a strong limitation to vertical resolution, which is inversely proportional to the total normal baseline aperture of the vertical array [RM00]. The look angle varies approximately from $20^{\circ}$ to $45^{\circ}$ from near to far range for the imaged swath, resulting in the vertical resolution to vary globally from $25 \mathrm{~m}$ to $300 \mathrm{~m}$. In the case of SHR the ratio between imaged ice thickness and tomographic resolution is low enough in most of the scene and the observed scattering can be regarded as mainly superficial. Flights were performed along an oval racetrack, in order to acquire data from two opposite views. In particular, $N=8$ tracks have been processed for each view, the tracks naming corresponds to the flight direction with respect to cardinal directions: NENW tracks and SWSE tracks. Conventional 2D SAR focusing was performed with Time-Domain Back Projection algorithm. Initially, data have been focused taking into account no other topographic information than the average site elevation, since the only external DEM available acquired around the time of the surveys was the NSDIC LVIS DEM [lvi] (airborne LIDAR data, acquired on May 10, 2012), which shows limited coverage with respect to the imaged area. Data have been subsequently refocused taking into account the DEM estimated with the proposed procedure, which has been iterated three times. Tomographic focusing was achieved with Capon spectral estimator [GLM02], so as to cope with the lack of resolution that characterizes Fourier beamforming due to the tight baseline tube. Images are represented in a Cartesian system of coordinates $(x, y, z)$, defined as azimuth, ground range and elevation of a fictitious reference track, common to both opposite-side surveys. Elevation is expressed with respect to WGS84 ellipsoid. In Figure 1 the intensity of master images from NENW and SWSE surveys is shown. It clearly appears how the overall scene lacks of robust features to be exploited by a classic radargrammetric approach. Moreover, some of the features observable may be related to subsurface phenomena. In Figure 2 vertical sections in the $x-z$ plane for NENW and SWSE views are illustrated before and after the application of the proposed methodology. LIDAR DEM is superimposed for comparison. The surface layer can be identified as corresponding to the upper boundary of Capon tomographic profiles. Before correcting rotations, residual azimuth varying low-pass undulations can be observed, as they hinder from retrieving the same absolute DEM in georeferenced coordinates from both views. For some locations these undulations are quite strong, as a consequence of the high sensitivity to error propagation of the estimates given by the small total normal baseline aperture of the experiment, as shown in Equation (2). The effectiveness of the proposed approach can be qualitatively appreciated observing the good superposition of the profiles corresponding to the two views after correcting for residual rotations, as well as a good agreement with LIDAR. It is worth remarking that profiles are represented in a georeferenced coordinate system, in this case a UTM grid centered and rotated so as to be aligned with the local coordinate system of the tomographic experiment, with elevation expressed with respect to WGS84 ellipsoid. It can be observed that rotations have been compensated to a great extent. Minor differences still persist as resulting from algorithmic errors and differences between NENW and SWSE profiles due to differences in vertical resolution values for the same spatial locations. In particular, the average DEM error after correction is quite well fitted with a Gaussian distribution of about mean $1 \mathrm{~m}$ and standard deviation $5 \mathrm{~m}$.

\section{CONCLUSION}

In this paper a new methodology to estimate the absolute DEM of a scene has been proposed. This methodology combines the advantages of precise estimation given by multi-baseline interferometric SAR processing with precise stereo plotting given by opposite-side radargrammetry. The procedure has been validated on a dataset in which there is absence of robust features for the application of conventional radargrammetry, namely land ice data from ESA IceSAR 2012 campaign. A good agreement has been obtained with external LIDAR data, proving the validity of the proposed methodology. Moreover, the procedure has been proven to work starting from few topographic information about the imaged scene and without exploiting any control point. Further work concerns open issues such as the definition of the best metric to be used in the matching step of tomographic profiles, the investigation of multi-squint interferometric processing [RPM06] to reduce azimuth varying low-pass undulations that affect the estimated topography and a theoretical error analysis of the proposed methodology.

\section{REFERENCES}

[CP10] K. M. Cuffey and W. S. B. Paterson. The Physics of Glaciers. Academic Press, 2010.

[DNKvdW13] J. Dall, U. Nielsen, A. Kusk, and R.S.W. van de Wal. Ice flow mapping with P-band SAR. In Geoscience and Remote Sensing Symposium (IGARSS), 2013 IEEE International, pages 251254, July 2013.

[GLM02] F. Gini, F. Lombardini, and M. Montanari. Layover solution in multibaseline SAR interferometry. Aerospace and Electronic Systems, IEEE Transactions on, 38(4):1344-1356, 2002.

[GTMdR11] G. Gatti, S. Tebaldini, M. Mariotti d'Alessandro, and F. Rocca. ALGAE: A Fast Algebraic Estimation of Interferogram Phase Offsets in SpaceVarying Geometries. Geoscience and Remote Sensing, IEEE Transactions on, 49(6):2343-2353, June 2011.

[lvi] Blair, J. B. and M. Hofton. 2010, updated 2014. IceBridge LVIS L2 Geolocated Surface Elevation Product. [GL2012]. 
Boulder, Colorado USA: NASA DAAC at the National Snow and Ice Data Center.

[MFP11] S. Meric, F. Fayard, and E. Pottier. A Multiwindow Approach for Radargrammetric Improvements. Geoscience and Remote Sensing, IEEE Transactions on, 49(10):3803-3810, Oct 2011.

$\left[\mathrm{PEB}^{+} 14\right]$ S. Perna, C. Esposito, P. Berardino, A. Pauciullo, C. Wimmer, and R. Lanari. Phase Offset Calculation for Airborne InSAR DEM Generation Without Corner Reflectors. Geoscience and Remote Sensing, IEEE Transactions on, PP(99):1-14, 2014.

$\left[\mathrm{RHJ}^{+}\right.$00] P.A. Rosen, S. Hensley, I.R. Joughin, Fuk K.Li, S.N. Madsen, E. Rodriguez, and Richard M. Goldstein. Synthetic aperture radar interferometry. Proceedings of the IEEE, 88(3):333-382, March 2000.

[RM00] A. Reigber and A. Moreira. First demonstration of airborne SAR tomography using multibaseline L-band data. Geoscience and Remote Sensing, IEEE Transactions on, 38(5):2142-2152, 2000.

[RPM06] A. Reigber, P. Prats, and J.J. Mallorqui. Refined estimation of time-varying baseline errors in airborne SAR interferometry. Geoscience and Remote Sensing Letters, IEEE, 3(1):145-149, Jan 2006.

[TMdBP13] S. Tebaldini, M. Mariotti d'Alessandro, F. Banda, and C. Prati. Tomographicquality phase calibration via phase center double localization. In Geoscience and Remote Sensing Symposium (IGARSS), 2013 IEEE International, pages 89-92, July 2013. 